\title{
Characterization of Non-linear Inductors Including Thermal Effects for Power Applications
}

\author{
D. Scirè ${ }^{1}$, S. Rosato ${ }^{1}$, G. Lullo ${ }^{1}$, and G. Vitale ${ }^{2}$ \\ ${ }^{1}$ Departement of Engineering, University of Palermo, Viale delle Scienze, Ed. 9, 90128 Palermo, Italy \\ e-mail: daniele.scire@unipa.it, samuele.rosato@libero.it, giuseppe.lullo@unipa.it \\ ${ }^{2}$ Institute of High Performance and Networking (ICAR), National Research Council (CNR), Palermo, Italy \\ e-mail: gianpaolo.vitale (at) icar.cnr.it
}

\begin{abstract}
This paper focuses on the behavior of non-linear inductors employed in DC/DC switching converters considering the effects due to variations of both current and temperature. The characterization of the inductors is performed by a buck converter operated to stimulate the inductor under test by a constant current with superimposed a large signal. The measurements are carried out for several temperature values of the inductor. Data are automatically collected by a novel virtual instrument developed by the authors in LabVIEW $^{\circledR}$ environment, aiming to obtain a polynomial based analytical model. The deep knowledge of the inductor model allows its design to be optimized improving the power density of the converter.
\end{abstract}

\section{Key words}

Inductor modelling, partial saturation, roll-off region, losses and temperature rise, $\mathrm{DC} / \mathrm{DC}$ converter.

\section{Introduction}

DC/DC converters in buck and boost configuration are widely used in many industrial applications. These circuits use the inductor as an energy reservoir, which is energized by the voltage source; the stored energy is transferred to the load operated at constant voltage thanks to an output capacitor. The optimization of the inductor in the converter design gives advantages in terms of power density increase and cost reduction; for this reason, this topic is becoming crucial in the design of modern power converters. In fact, a reduction of the value of the inductance lessens both the weight and the size. This can be achieved by operating the converter at a higher switching frequency, but causes an increase of switching losses in the power devices, which require larger heatsinks, and in the magnetic material. Reducing the inductance, a higher ripple current results, the magnetic material is better exploited, the off-on switching is performed with a lower current but the peak of the current may lead the inductor operating point outside of the linear region. This constraint requires a deep knowledge of the model of the inductor since it becomes non-linear and temperature dependent. This issue is of great interest both for scientists and industry due to economic reasons tied both to the initial cost of the converter and to the reduced payback time of the equipment [1]. The value of the inductor in power converters is often considered constant with the current both for static and State Space Averaging analysis $[2,3]$; recently some papers dealing with the use of inductors working in partial saturation [4-7] have been published. In $[8,9]$ the thermal model has been considered as well. In these cases, the inductor is analyzed under current variation from a minimum to a maximum during the switching period, thus including the saturation region. However, saturation implies a more complex control of the converter. Inductor cores are generally made by ferrite and iron powder, the latter being affected by a sharp transition between nominal and saturation values. A particular attention is required to avoid a sudden increase of current due to the inductance decrease.

In this scenario, the lack of specific information on datasheets about saturation curve and temperature behavior discourages the use of partially saturated inductors, although there is a keen interest in exploiting partial saturation in some magnetic materials such as ferrite. In the literature, there are examples of soft ferrite based wireless power transmission systems [10] and of innovative electric traction motors using ferrite as magnetic material [11].

In order to properly design the converters, it is necessary to increase the knowledge of magnetic material behavior as the current changes; for this purpose, there is a need to develop new models. In [12] a model, involving temperature as a variable, has been introduced for the description of the temperature-dependent magnetic hysteresis of soft ferrite material. In [13] is shown that every global model of inductance saturation curve, which involves a large-amplitude current ripple condition, is associated with a specific inductor temperature.

The authors have proposed an inductance measurement system by varying only the current level in [5] and [14]. In this contribution, the system is improved to match the simulation and the experimental data including thermal effects. As a matter of fact, during operation, the temperature of the magnetic material varies changing the inductor value. 
This paper proposes a new automatic measuring system which can acquire many curves of inductance vs. current, each one for a different temperature.

The system uses a simple DC/DC buck converter, in which its inductor represents the inductor under test, with an active load and a readout virtual instrument implemented in LabVIEW ${ }^{\circledR}$ programming environment. The buck converter is controlled to supply the inductor under test with a DC current with superimposed ripple, the current waveform is sampled and processed by LabVIEW ${ }^{\circledR}$.

This paper is organized as follows: in section two the behavior of the inductors for high current and temperature is analyzed. Section three deals with the electronic circuit used for inductor characterization. In section four the LabVIEW $^{\circledR}$ measurement virtual instrument is described while the experimental rig is presented in section five. Experimental results are finally given in section six.

\section{Inductor Characterization}

\section{A. Moderate Saturation and Temperature Effect}

The saturation of the magnetic core of inductors used in DC/DC converter applications causes a variation of the inductance (L) with the current (I). Figure 1 shows the variation of inductance of a set of commercial inductors vs. DC current [15]. The single L vs. I curve, however, is associated to a specific temperature value $(\mathrm{T})$.

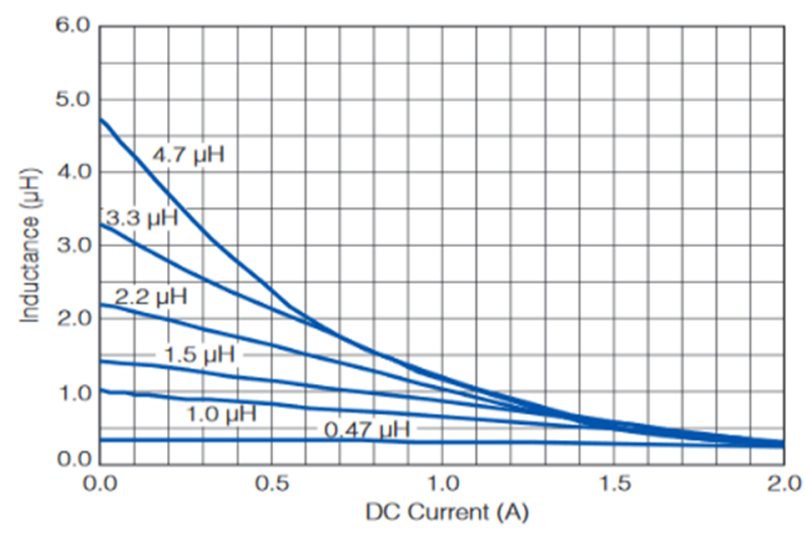

Figure 1: Dependence of inductance vs DC current for some commercial inductors (ELGTEAXXXNA) [15].
The use of inductors with cores made of soft magnetic materials means that component characteristics depend on the temperature behavior of the core material. So magnetic properties, like permeability and saturation, of the core material will change with temperature [16]. As an example, Panasonic has designed a family of power choke coils (PCC) with high thermal stability and with a smaller volume than ferrite [17], whose performance comparison with ferrite is shown in Figure 2. Ferrite type PCC shows that the saturation curves are temperature dependent, while the Panasonic PCC M0754 is temperature independent because of non-magnetic saturation.

If the inductor operates with a small ripple of the current, a small variation of the inductance value is expected; however, a larger current ripple around the DC point grants better exploitation of the magnetic material preventing the oversize of the inductor. On the other hand, larger current ripple provokes the variation of the inductance during the switching period.

The inductance value depends also on the temperature of the core; a greater temperature emphasizes the saturation effect of the inductor and must be considered in the design process. The inductor temperature depends in turn on circuit design and, due to the power loss, is strictly related to the DC operating point.

Thus, for a proper model of the non-linear behavior of the inductors, it is necessary to provide information about the effect of temperature presenting a set of curves L vs. I which differ from each other in the temperature of the inductor. Therefore, a curve set like the one showed in Figure 1, given at a single temperature, cannot be used in a great ripple scenario requiring a dedicated characterization system like the one proposed in this paper.

\section{B. The inductors under test}

The ferrite inductor chosen for the characterization is the Panasonic ELC18B471L $\left(470 \mu \mathrm{H}, \mathrm{I}_{\mathrm{DC}}=1.6 \mathrm{~A}\right)$; the rated current of these inductors represents the smaller value between either the current value when the inductance drops $10 \%$ down from its initial point or when the average temperature of coil interior rises $45^{\circ} \mathrm{C}$. Except for these data, no further information about saturation curves or effects of temperature over inductance is given in the datasheets of the components; therefore a characterization of these inductors would allow designers to use them also in the moderate saturation region.

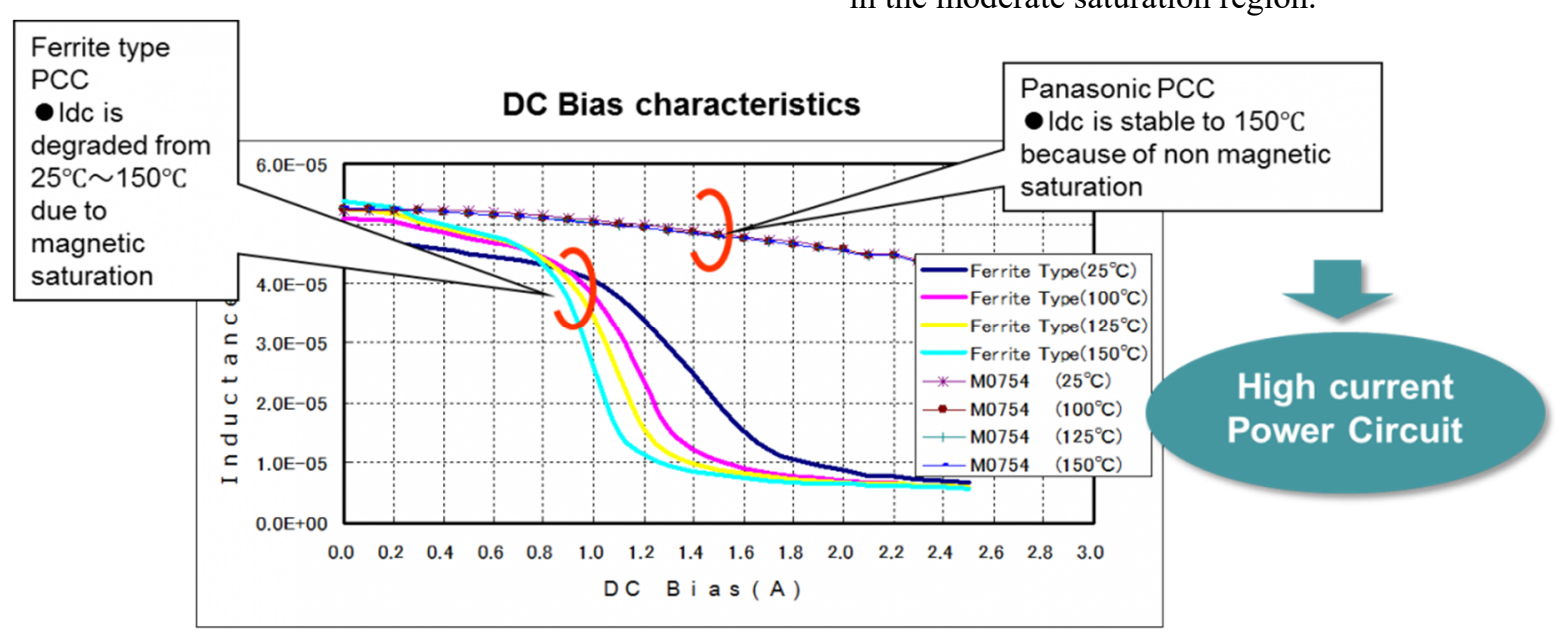

Figure 2: DC Bias characteristic for different temperatures (Ferrite and M0754) [17]. 


\section{Characterization}

Experimental data came from an automatic measurement virtual instrument developed in LabVIEW ${ }^{\circledR}$ environment; the system automatically produces an L vs. I curve for a given maximum current. For each curve, a temperature measurement is made by a thermocouple applied to the case of the inductor. In this way, it is possible to produce a family of $\mathrm{L}$ vs. I curve, each one for a given temperature.

To achieve uniform heating of the material, the inductor was immersed in a beaker filled with distilled water, which is preheated by a hot plate (Figure 3 ).

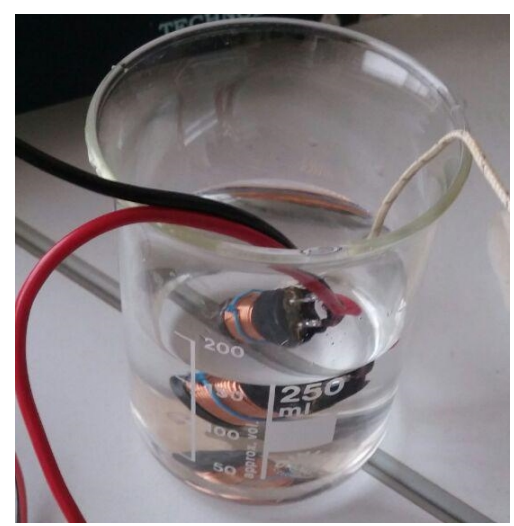

Figure 3: Inductor under test immersed in hot distilled water.

The heat capacity of the water, moreover, allows to maintain the temperature of the core constant during the measurement; without the water, in fact, the temperature would change when the current rises.

This method for temperature stabilization was considered appropriate because it proved not to introduce any significant differences in the waveforms' aspect and in the measured value of inductance.

\section{The measurement system}

Usually, the commercial systems are not able to test inductors in operating conditions reproducing their use in a power converter, i.e. with high DC current and a large ripple superimposed. For this reason, a dedicated system based on a DC/DC buck converter has been set up. An algorithm that allows a configurable number of automated measurements of inductance has been implemented. These tests are performed on the same inductor as the current changes, in order to plot the inductance $L$ versus the inductor current $I$ at a fixed temperature.

The PWM modulation of a square wave signal, produced by the waveform generator, is used to control the switch of the buck converter. The output voltage $\left(\mathrm{V}_{\mathrm{o}}\right)$ applied to the active load is regulated by adjusting the duty cycle (D) of the driving signal according to the equation:

$$
V_{o}=\frac{t_{O N}}{T_{s}} V_{d}=D V_{d}
$$

where $V_{d}$ is the DC input voltage and $T_{o n}$ is the ON time of the power device with switching period $T_{s}$.

Changing, step by step, the value of current absorbed by the active load, it is possible to vary the DC current of the inductor under test.

By applying a suitable switching frequency to the converter switch, it is possible to obtain a triangular waveform of the inductor current. The slope of the current ramps is calculated from the waveform data acquired by an oscilloscope (Figure 4).

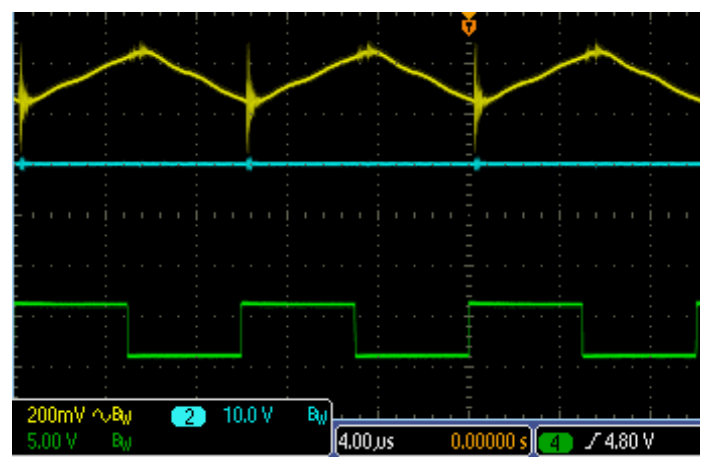

Figure 4: Experimental waveforms measured in the DC/DC converter with small ripple: inductor current (yellow), output voltage (azure), switching control signal (green).

The measurement is carried out by exploiting the inductor constitutive equation rewritten as:

$$
L=\frac{V_{L}}{\frac{d I}{d t}}
$$

where $I$ and $V_{L}$ are the current and the voltage across the inductor of inductance $L$.

The final result of inductance measurements is processed and used as a value to plot the L vs. I curve.

For small current variations, the value of $L$ can be considered constant. The progressive increase of the average current on the inductor moves its operating point from the linear region to the partial saturation region, resulting in a decrease in the inductance value.

The DC bias current, the frequency and the duty-cycle of the switching element can be controlled by a virtual instrument developed in LabVIEW, which also takes care of data acquisition and post elaboration.

The scheme shown in Figure 5 represents the structure of the electronic circuit designed and implemented in this work.

The circuit consists of the following blocks:

- $\mathrm{DC} / \mathrm{DC}$ buck converter;

- current sensing;

- active load.

\section{A. The Converter used as test system}

The core of the measurement setup is a simple buck converter that works in continuous conduction mode (CCM).

The converter switch is the IRFP150 power MOSFET. The control signal for the high-side MOSFET is provided by the driver IR2101 (not shown in Figure 5) and its bootstrap circuit.

The closing and opening times of the switch and therefore the average voltage on the load are controlled by a square wave of frequency $100 \mathrm{kHz}$. The free-wheeling diode is the MURB820PBF, chosen for its low switching losses, optimized stored charge and low recovery time.

The output capacitor is an electrolytic capacitor of $100 \mu \mathrm{F}$ and its value has been chosen to limit the load voltage ripple. 


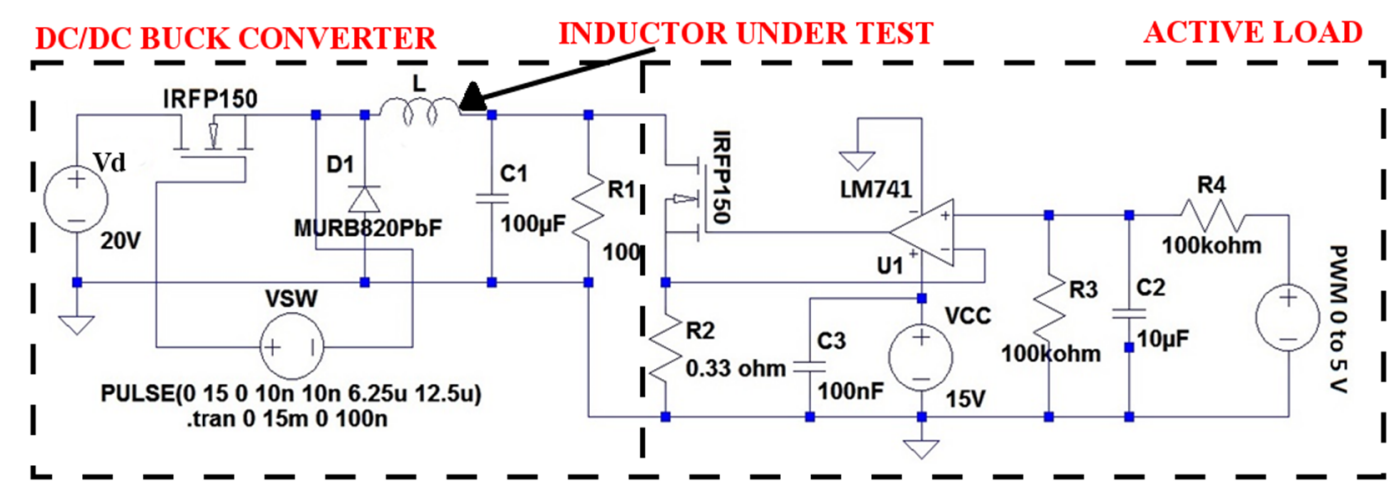

Figure 5. Schematic of the electronic circuit of the test board.

\section{B. Current Sensing}

A Hall-effect current transducer $L A$ 55-p converts the current flowing through the inductor into a voltage across the leads of a sense resistor.

This voltage is acquired by an oscilloscope probe, as shown in Figure 6.

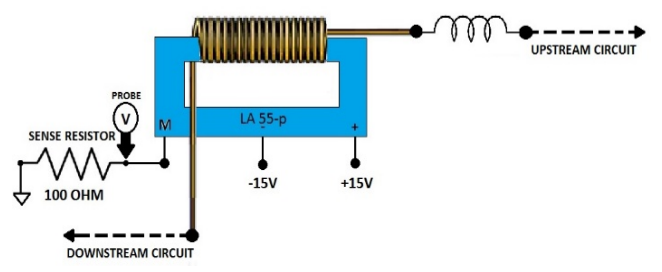

Figure 6. Current sensing system.

\section{Active Load}

In order to measure the inductance with different values of the DC bias current, a constant current sink is placed after the LC filter as an active variable load.

The circuit is composed of a MOSFET IRFP150, whose drain is connected to the output of the buck converter.

Three $1 \Omega, 5 \mathrm{~W}$ resistors, arranged in parallel, are connected on the source thus being equivalent to a load resistor of $0.33 \Omega$ with a $15 \mathrm{~W}$ dissipation capability.

The current sunk by the MOSFET varies according to a reference voltage applied to the OPAMP LM741 that implements, together with the MOSFET, a voltage-tocurrent converter. The OPAMP drives the gate of the MOSFET to make the voltage across the load resistor equal to the reference voltage. In this way is possible to impose the current absorbed by the circuit simply varying the reference voltage.

The reference voltage for the OPAMP is obtained as the average value of a PWM signal, provided by an Arduino Uno board, filtered by a low pass filter consisting of two $100 \mathrm{k} \Omega$ resistors and a $10 \mu \mathrm{F}$ electrolytic capacitor. The PWM switching frequency is $160 \mathrm{kHz}$.

In the right part of Figure 5, it is possible to see the circuit just described.

\section{Virtual Instrument}

The development of the measurement virtual instrument has been driven by the need of creating a user-friendly environment that allows making quick and accurate automatic measurements.

The implemented program allows the user to interact with the computer and the controlled instrumentation. The software provides the user with a graphical "virtual instrument" for setting the measurement parameters and manipulating the acquired data. The front panel of the virtual tool is shown in Figure 7.

It consists of several potentiometers, pushbuttons, indicators and virtual LEDs and a graph. The operator can set the input parameters and instantaneously check the parameters that will be automatically set at each measurement. After the acquisition, data elaboration follows, then the results obtained are shown on a graph to the operator. The data can be exported as an Excel spreadsheet or as an image file for later post-processing.

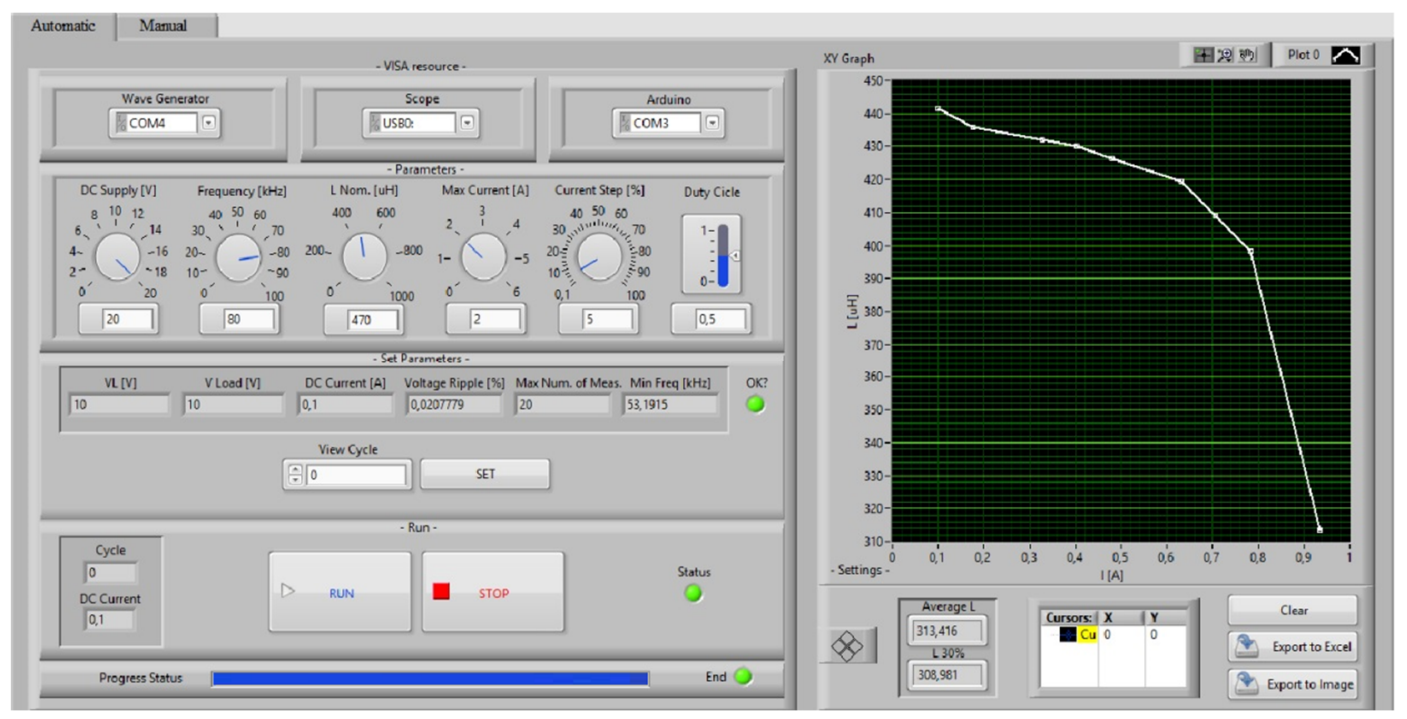

Figure 7. LabVIEW virtual instrument front panel. 
The description of the implemented algorithm is shown in the flowchart in Figure 8.

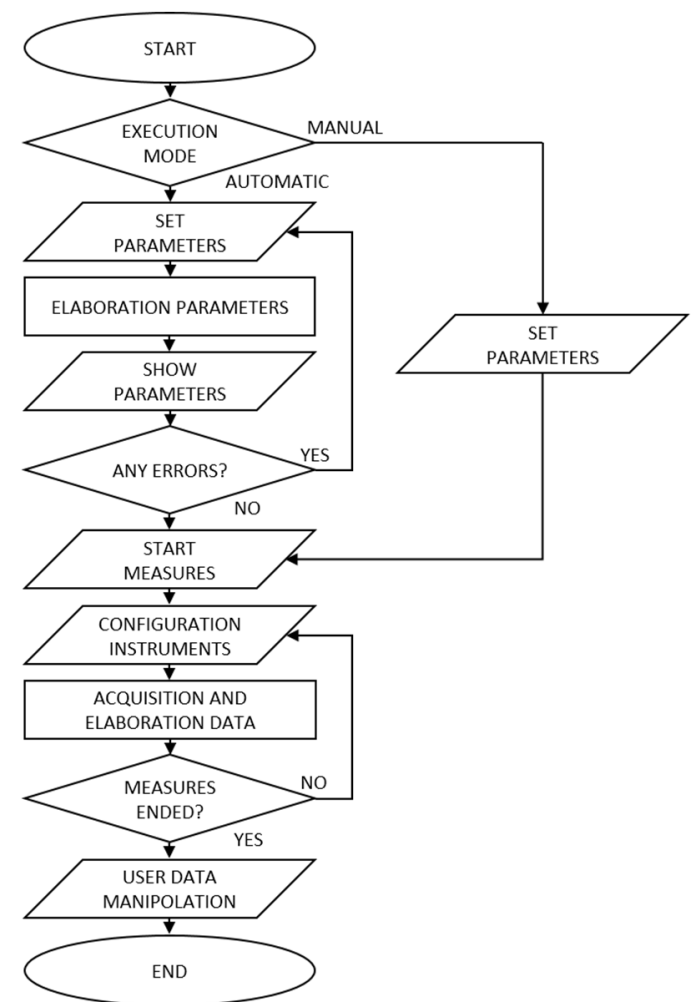

Figure 8. Flowchart of the algorithm implemented for the test.

The block diagram of LabVIEW software is divided into several elements made up of various internal nested structures that interact with each other.

The first block consists of the outer structure of the program, which encloses the entire block diagram. It is implemented by a case structure, i.e. a case selector that can contain one or more sub-diagrams that can be executed when the structure is running.

The blocks of the diagram follow the "event structure": operations are only performed when the value of a control is changed. Event management is the best solution for optimizing application resources because, regardless of other conditions, the code will only run when it is needed [18].

Once the program is running, the operator can choose between two execution modes by selecting one of the two tabs on the top left corner in the front panel. The execution can be manual or automatic.

The two execution modes are quite similar to each other and mainly carry out similar measurement procedures.

\section{A. Manual Mode}

The manual mode allows performing measurements on the unknown inductor, of which the inductance value, the nominal current, or both are not known in advance. In this case, it is advisable to proceed by acquiring one point at a time starting from the smaller current values that can be set by the Arduino Uno system, until a decrease of the inductance value is detected. In addition to the circuit parameters, it is possible to set the maximum current value. This current value is chosen based on empirical considerations performed by the operator so as not to damage the component under test.
This mode of operation is slower but is the preferred one for checking unknown inductors.

\section{B. Automatic Mode}

The automatic mode presupposes the knowledge of the rated value of inductance and maximum DC bias current of the inductor. In this case, it is possible to set the parameters and choose the span of inductor DC bias current between the measures; in this way, the maximum number of inductance measures is set. Reducing the span, the number of measurements increases leading to an increase of the time spent to complete the set. Vice versa, the increasing of the span reduces the time spent for the entire collection, but the L vs. I curve has a smaller number of points.

\section{The Test Rig}

The test rig is composed of: a DC 6A power supply; the $\mathrm{DC} / \mathrm{DC}$ converter; the inductor under test; the active load; a wave function generator; a personal computer; a hot plate for heating the inductor; a digital multimeter equipped with a thermocouple; an oscilloscope equipped with voltage probe to acquire the driving signal and a current transducer to sense the current in the inductor. The main components of the experimental rig are summarized in Table I.

Table I. The test rig.

\begin{tabular}{|l|l|l|}
\hline Supplier & Function & Model \\
\hline HQ Power & Power Supply & PS23023 \\
\hline TTi & Signal Generator & TGA1241 \\
\hline $\begin{array}{l}\text { Chemat } \\
\text { Technology Inc. }\end{array}$ & Hot Plate & KW-4AH \\
\hline Tektronik & Oscilloscope & MSO 2024B \\
\hline Lafayette & Digital Multimeter & UT70B \\
\hline
\end{tabular}

\section{Experimental Results}

Using the proposed measurement system, a family of isothermal inductance curves has been obtained for the inductor under test. The aggregate data relative to the $470 \mu \mathrm{H}$ inductor are shown in a 3-D surface in Figure 9.

For currents lower than $2.5 \mathrm{~A}$ the curves are similar and there is no significant temperature effect on the value of inductance. The same observation is valid for currents higher than $4.5 \mathrm{~A}$; at this point, the inductance drops to the $20 \%$ of the nominal value and the inductor is in deep saturation region.

In the roll-off region, between $2.5 \mathrm{~A}$ and $4.5 \mathrm{~A}$, it can be noted that for higher temperatures the inductance curves shift downwards, indicating a smaller inductance.

Figure 10 shows the same data of Figure 9 arranged to highlight the variation of the inductance as a function of temperature for different DC currents. For currents lower than $2.5 \mathrm{~A}$ the curves are quite flat, showing no variation of inductance for increasing temperatures; the curves in the roll-off region, instead, show a constant slope. As the inductor approaches the deep saturation region, the temperature minimizes his effect on the inductance variation. 


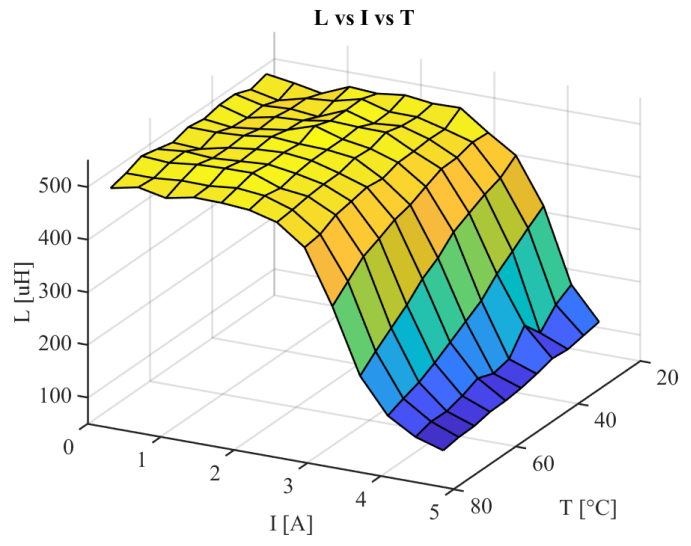

Figure 9. Saturation surface for different temperatures and currents.

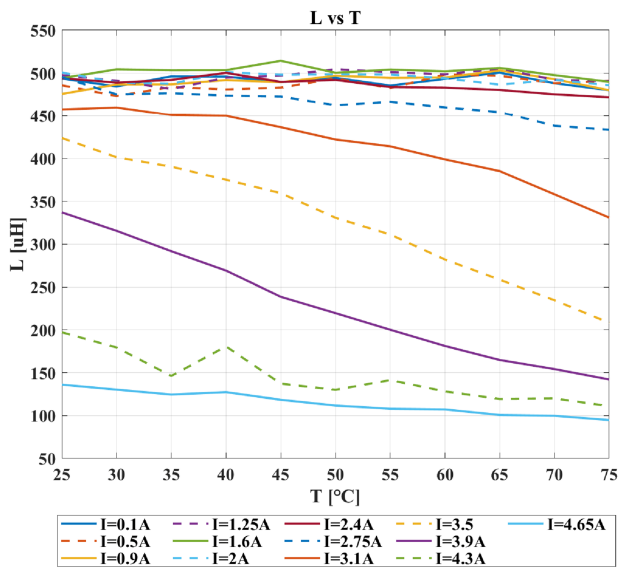

Figure 10. Inductance vs. Temperatures at different DC currents.

In Figure 11, a single saturation curve is represented; an error bar is set in each data point of the curve. These bars represent the standard deviation of the measure. The calculated relative standard deviation $\boldsymbol{\sigma}^{*}$ is 0.0075 ; this value is obtained iterating the measuring process multiple times while maintaining the same operating conditions.

By the numerical values acquired with the proposed system, it is possible to obtain a continuous function, to be used in a simulator as in [5], to reproduce the inductance behavior. In particular, the inductance versus the current can be approximated by a $5^{\text {th }}$ order polynomial function as shown in Figure 12, whereas the inductance versus temperature curve shows a linear trend as sketched in Figure 13.

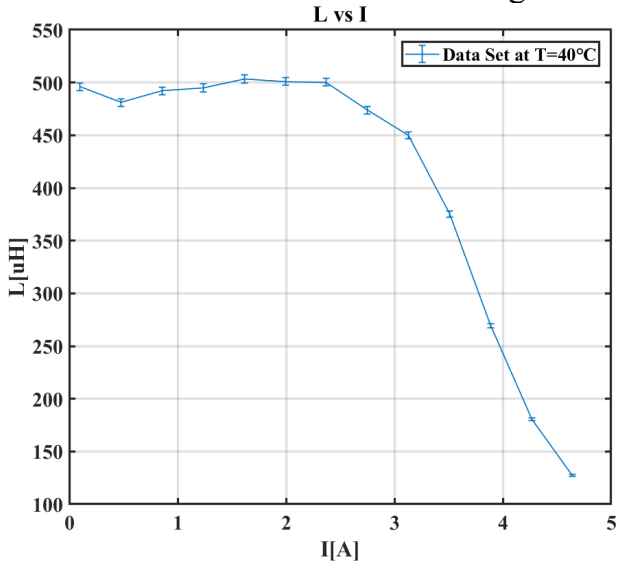

Figure 11: Single saturation curve and its error bars.

\section{Conclusion}

A measurement system for inductance characterization has been implemented using a DC/DC buck converter and a LabVIEW virtual instrument developed to the purpose. The behavior of a power inductor has been analyzed for a range of DC operating points. The effect of temperature in the non-linear $\mathrm{L}$ vs. I curve has been verified experimentally.

It has been found that the temperature effect is more evident when the inductor operates in the roll-off region, while in the weak saturation and in deep saturation this effect is quite limited.

The experimental data provide a set of isothermal inductance saturation curves. As expected, the operation with large currents signal highlights the variation with temperature of the inductance. These curves can be used in the design process for applications which involve inductors operating in moderate saturation region since it is possible to obtain a model based on polynomials including the temperature as well.

It has been underlined that the isothermal curves can be approximated by fifth-degree polynomials and that the inductance versus temperature curves are quite linear for given values of the current.

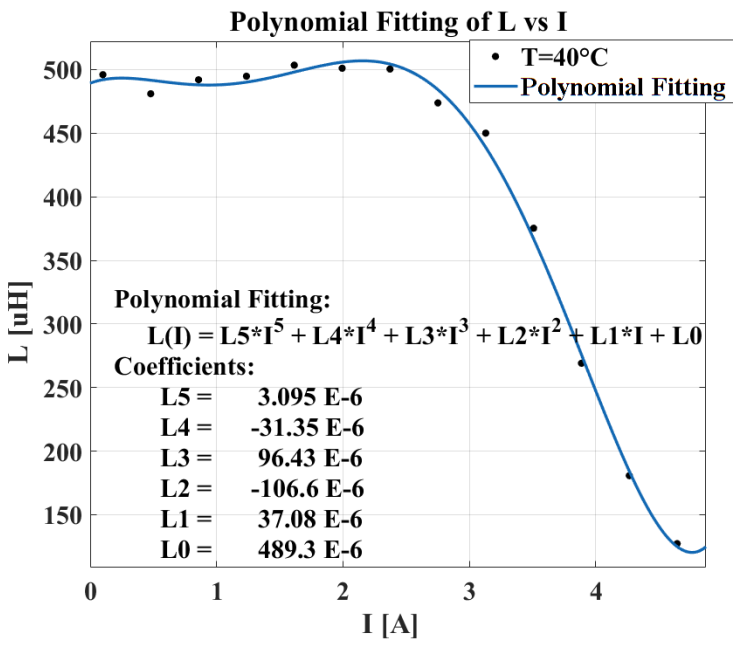

Figure 12. Polynomial fitting of an L vs. I curve.

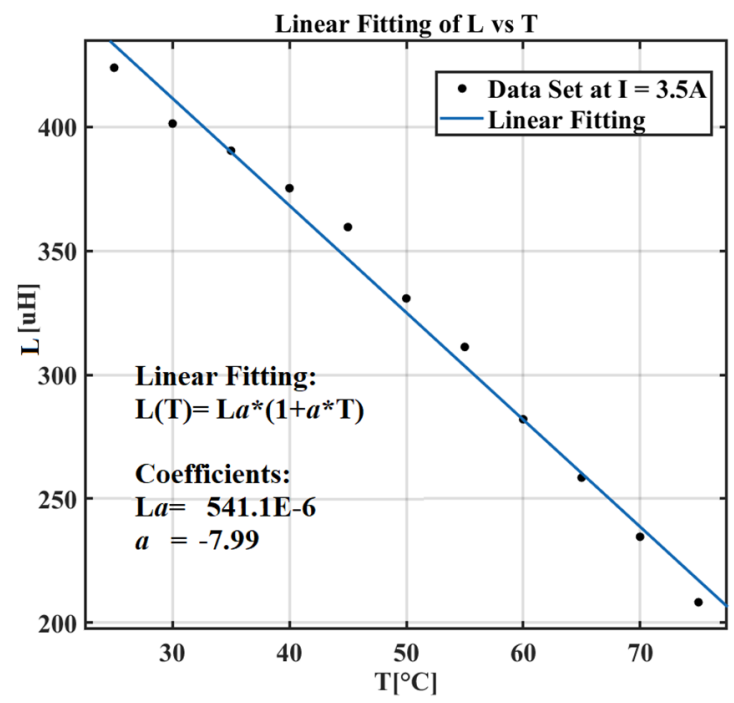

Figure 13: Linear fitting of an L vs. I curve. 


\section{References}

[1] G. Vitale, «Energy saving by power electronics: toward a new concept of renewble source» Renewable Energy \& Power Quality Journal, vol. May 2016, n. 14, pp. 160001160010, 2016.

[2] N. Mohan, T. M. Undeland and W. P. Robbins, Power Electronics: Converters, Applications, and Design, 3rd Edition, Wiley, 2002.

[3] R. W. Erickson and D. Maksimovic, Fundamentals of Power Electronics, Second Edition, Springer, 2001.

[4] G. Di Capua and N. Femia, «A Novel Method to Predict the Real Operation of Ferrite Inductors Witth Moderate Saturation in Switching Power Supply Applications» IEEE TRANSACTIONS ON POWER ELECTRONICS, vol. 31, n. 3, 2016

[5] G. Lullo, D. Scirè and G. Vitale, «Non-linear inductor modelling for a DC/DC Buck converter» Renewable Energy and Power Quality Journal (RE\&PQJ), n. 15, 2017.

[6] L. Milner and G. A. Rincòn-Mora, «Small saturating inductors for more compact switching power supplies» IEEJ Transactions on Electrical and Electronic Engineering, vol. 7, n. 1, pp. 69-73, 2012.

[7] D. Scirè, G. Vitale and G. Lullo, «Design and realization of a DC/DC converter with Inductor in partial saturation» in 49th Annual Meeting of Associazione Societa' Italiana di Elettronica (SIE), Palermo, Italy, 2017.

[8] K. Detka, K. Górecki and J. Zarębski, «Modeling Single Inductor DC-DC Converters With Thermal Phenomena in the Inductor Taken Into Account» IEEE TRANSACTIONS ON POWER ELECTRONICS, vol. 32, n. 9, pp. 7025-7032, 2017.

[9] K. Górecki and M. Godlewska, «Electrothermal Model of Ferromagnetic Cores» PRZEGLAD ELEKTROTECHNICZNY, vol. 91, n. 3, pp. 161-165, 2015.

[10] W. Lee, Y.-K. Hong, J. Park, J. Lee, I.-S. Baek, N.-P. Hur, W.-M. Seong and S.-O. Park, «A Simple Wireless Power Charging Antenna System: Evaluation of Ferrite Sheet» IEEE TRANSACTIONS ON MAGNETICS, vol. 53, n. 7, 2017.
[11] T. Fukami, K. Motoki, R. Kirihata, K. Shima, M. Koyama, T. Mori and M. Nakano, «An Electromagnet-Assisted Ferrite Magnet Motor» IEEE TRANSACTIONS ON MAGNETICS, vol. 53, n. 11, 2017.

[12] N. Duan, W. Xu, S. Wang, J. Zhu, R. Qu and a. S. Jia, «A Temperature-Dependent Hysteresis Model for Soft Ferrites» IEEE TRANSACTIONS ON MAGNETICS, vol. 53, n. 6, 2017.

[13] G. D. Capua, N. Femia, K. Stoyka, M. Lodi, A. Oliver e M. Storace, «Ferrite Inductor Models for Switch-Mode Power Supplies Analysis and Design» in 14th International Conference on Synthesis, Modeling, Analysis and Simulation Methods and Applications to Circuit Design (SMACD 2017), Giardini Naxos - Taormina, Italy, 2017.

[14] S. Rosato, G. Vitale e G. Lullo, «Characterization of inductors in partial saturation for SMPS applications» in 49th Annual Meeting of Associazione Societa' Italiana di Elettronica (SIE), Palermo, Italy, 2017.

[15] Panasonic Corporation, «Panasonic Chocke Coils - Power Inductors / Multilayer type - Type: ELGTEA» 1 September 2012. [Online]. Available: https://www.mouser.it/pdfdocs/Panasonic_ELG_TEA_Ind uctors.PDF.

[16] EPCOS, «Inductors - General Technical Information» October 2008. [Online]. Available: https://en.tdk.eu/download/531382/3c79b910253035d31f5 97fcd60b5a919/pdf-generaltechnicalinformation.pdf.

[17] Panasonic Industry Europe, «Power Choke Coils for Automotive Applications» [Online]. Available: https://eu.industrial.panasonic.com/node/2539/powerchoke-coils-automotive-applications.

[18] National Instruments Corporation, «Event-Driven Programming in LabVIEW» 20 July 2008. [Online]. Available: http://www.ni.com/white-paper/3331/en/. 